\title{
Diagnostic Utility of Prostein, Uroplakin II and SATB2 for Diagnosing Carcinoma of Unknown Primary Origin: A Systematic Immunohistochemical Profiling
}

\author{
KUNIO MOCHIZUKI, MASATAKA KAWAI, TORU ODATE, IPPEI TAHARA, TOMOHIRO INOUE, \\ KAZUNARI KASAI, TADAO NAKAZAWA, RYOHEI KATOH and TETSUO KONDO \\ Department of Pathology, School of Medicine, University of Yamanashi, Chuo, Japan
}

\begin{abstract}
Background/Aim: Immunohistochemistry was used to evaluate 600 carcinomas of major histological types from various organs to determine the tissue distributions of the novel markers prostein, uroplakin II and SATB2. Materials and Methods: We retrieved 30 cases from 20 different carcinomas of systemic organs. Results: All prostate adenocarcinomas were immunopositive for prostein, and its reactivity was consistently diffuse. There was faint labeling of prostein in few cases of the 570 non-prostatic carcinomas. Uroplakin II was immunopositive in 53\% and $60 \%$ of urothelial carcinomas (UC) of the bladder and the ureter, respectively. There was focal and weak positivity of uroplakin II in a few cases of non-urinary tract carcinomas. SATB2 was frequently positive in adenocarcinomas of the digestive organs, and was also expressed in a minority of the non-colorectal adenocarcinomas. Conclusion: Prostein and uroplakin II are immunohistochemical biomarkers of prostate adenocarcinomas and UCs of the urinary tract.
\end{abstract}

Approximately $3-5 \%$ of new malignant diagnoses are classified as carcinomas of unknown primary origin (1). This type of diagnosis is defined by histologically-confirmed metastatic carcinoma in the absence of clinical, radiographic, or pathologic identification of a primary site. Although patients with unknown primary carcinomas are treated with surgery, radiation therapy or chemotherapy according to general carcinoma treatment guidelines, there are specific treatment guidelines for a primary tumor when identified that are based on the oncological principles established for each primary tumor type (2). In the current era of tissue-specific

Correspondence to: Kunio Mochizuki, MD, Ph.D., Department of Pathology, School of Medicine, University of Yamanashi, 1110 Shimokato, Chuo, Yamanashi, 409-3898, Japan. Tel: +81 552739529, Fax: +81 552739534, e-mail: kuniom@yamanashi.ac.jp

Key Words: Prostein, uroplakin II, SATB2, immunohistochemistry. therapeutic strategies, it is important to establish a tumor's origin, even when dealing with metastatic disease, because targeted treatment can potentially provide better symptomatic relief and prolonged survival (3). For example, outcomes for metastatic colorectal cancer patients have improved by treating patients with anti-epidermal growth factor receptor antibodies, particularly when combined with predictive biomarkers to select for patients lacking $R A S$ mutations (4). Consequently, finding a better way to identify a carcinoma's primary site could improve treatment outcome for carcinomas of unknown origin.

Although histomorphologic examination using hematoxylin and eosin (HE) stained slides can provide a pathological diagnosis of a tumor, an accurate diagnosis may be difficult in small biopsy specimens due to a variety of reasons such as scant tumor cells, lack of characteristic architecture in small biopsy specimens, artifacts in specimen preparations, and differentiation and heterogeneity of the carcinoma. Poorly differentiated carcinomas are particularly difficult to classify since they lack specific architectural or cytological features indicating histologic type. A diagnostic approach using immunohistochemistry is essential for evaluating biopsies of carcinomas of unknown origin. This study focused on the novel markers prostein, uroplakin II and special AT-rich sequence-binding protein 2 (SATB2). Prostein is a 553-amino acid protein identified by cDNA library subtraction and subsequent high-throughput microarray screening of prostate cancer, and it seems to be selectively expressed in both benign and malignant prostate epithelium (5). Uroplakin II is a $15-\mathrm{kDa}$ protein component of the urothelial plaques that enhances the permeability barrier and strength of the urothelium (6). The expression of uroplakin II is aberrant in urothelial carcinoma (UC) of the bladder, and thus, it may be a useful marker for the diagnosis of bladder carcinoma (6). SATB2 is a described transcriptional regulator that is involved in osteoblastic and cortical neuron differentiation and in skeletal development. Immunohistochemical studies have shown that SATB2 is not only expressed by normal and 
neoplastic osteoblastic tissues, but it is also strongly expressed by normal colorectal and appendiceal epithelium (7). Although there are a few studies examining the use of prostein, uroplakin II and SATB2 as markers for determining primary sites of carcinomas of unknown origin, these studies examined a limited number of carcinoma cases directed at a limited number of organs $(3,5-20)$.

In this study, immunohistochemistry was used to systematically evaluate 600 cases of 20 major histological types of carcinoma arising from 16 organs (larynx, esophagus, stomach, colon, thyroid, lung, liver, extrahepatic bile duct, gallbladder, pancreas, urinary bladder, ureter, prostate, breast, uterine corpus and ovary). We hoped to determine the tissue distributions of prostein (as a marker for prostatic adenocarcinoma), uroplakin II (as a marker for UC of the urinary tract) and SATB2 (as a marker for colorectal adenocarcinoma) and to examine the usefulness of these markers for determining the primary sites of carcinomas of unknown origin.

\section{Materials and Methods}

Patients. We collected 30 samples from each of the following primary tumor types: conventional squamous cell carcinomas (SCCs) of the larynx, SCCs of the esophagus, tubular adenocarcinomas of the stomach, poorly cohesive carcinomas of the stomach, ordinary adenocarcinomas of the colon, mucinous adenocarcinomas of the colon, papillary carcinomas (PCs) of the thyroid, adenocarcinomas of the lung, SCCs of the lung, classical hepatocellular carcinomas (HCCs) of the liver, adenocarcinomas of the liver, ordinary adenocarcinomas of the extrahepatic bile duct, ordinary adenocarcinomas of the gallbladder, ductal adenocarcinomas of the pancreas, infiltrating UCs of the urinary bladder, infiltrating UCs of the ureter, acinar adenocarcinomas of the prostate, invasive carcinomas of no special type (IC-NSTs) of the breast, endometrioid carcinomas (ECs) of the uterine corpus and clear cell carcinomas (CCCs) of the ovary obtained surgically at the University of Yamanashi Hospital. Two pathologists (K.M. and T.K.) independently reviewed HE stained slides blinded to the original pathological diagnosis. The Research Ethics Committee of Faculty of Medicine, University of Yamanashi approved this study (approval number 1664 and 1850).

Immunohistochemistry. Sections $4-\mu \mathrm{m}$ thick were cut from formalinfixed, paraffin-embedded tissue blocks that were dewaxed and rehydrated. Representative slides received immunohistochemical staining. Table I lists the primary antibodies used, their dilutions and their sources. Antigen retrieval was accomplished through heat treatment before performing the primary antibody reactions: autoclaving at $120^{\circ} \mathrm{C}$ for 10 min in Antigen Retrieval Solution pH9 (Nichirei Biosciences, Tokyo, Japan) for prostein and SATB2, and $120^{\circ} \mathrm{C}$ for $10 \mathrm{~min}$ in citrate buffer $\mathrm{pH} 6$ for uroplakin II. After inhibiting endogenous peroxidase, positive controls were used to perform the primary antibody reactions. N-Histofine Simple Stain MAX PO (MULTI) (Nichirei Biosciences, Tokyo, Japan) was used with diaminobenzidine as a chromogen and a light counterstain with hematoxylin to perform immunohistochemistry. Two pathologists (K.M. and T.K.) simultaneously reviewed immunostained sections using a double-headed light microscope. They scored the extent of
Table I. Antibodies.

\begin{tabular}{lccc}
\hline Antibody & & Source & Dilution \\
\hline Prostein & $10 E 3$ & Dako, Glostrup, Denmark & $1: 400$ \\
Uroplakin II & BC21 & Biocare Medical, & $1: 100$ \\
SATB2 & EPNCIR130A & Pacheco, CA, USA & Abcam, Cambridge, UK \\
\hline
\end{tabular}

immunohistochemical staining among the tumor cells as $0=0 \%$ reactive tumor cells, $1+=1 \%$ to $10 \%$ reactive tumor cells; score $2+=11 \%$ to $50 \%$ reactive tumor cells, and $3+=51 \%$ to $100 \%$ reactive tumor cells. Specimens assessed at $1+, 2+$ or $3+$ were defined as immunopositive and sections assessed at 0 as immunonegative.

\section{Results}

Table II shows the results of the immunohistochemical staining of prostein. Prostein expressed in only 3 types of carcinoma. Acinar adenocarcinoma of prostate samples had $3+$ expression in $100 \%$ of the cases (Figure 1A and B). However, liver adenocarcinoma had $1+$ expression in $1(3 \%)$ case, and IC-NST of breast samples had 2+ expression in 1 (3\%) case (Figure 1C and D). The sensitivity and specificity of prostein for the diagnosis of acinar adenocarcinoma of prostate origin were $100 \%$ and $99.6 \%$, respectively.

Table III shows the results of the immunohistochemical staining of uroplakin II. Uroplakin II expressed in 6 types of carcinoma. Infiltrating UC of the urinary bladder had 16 (53\%) positive cases: 4 at $1+, 7$ at $2+$ and 5 at $3+$ (Figure $2 \mathrm{~A}$ and $\mathrm{B})$. Infiltrating $\mathrm{UC}$ of the ureter had similar results with $18(60 \%)$ cases: 7,7 and 4 cases at $1+, 2+$ and $3+$, respectively. Ordinary adenocarcinoma of the colon, SCC of the lung, and EC of the uterine corpus had 1 (3\%) case each that scored $1+$, and classical HCC of the liver had $3(10 \%)$ cases that scored $1+$. The HCC cells exhibited uroplakin II immunoreactivity in the intracytoplasmic hyaline bodies characteristically and exclusively (Figure 2C and D). The sensitivity and specificity of uroplakin II for the diagnosis of UC of urinary tract origin were $57 \%$ and $99 \%$, respectively.

Table IV shows the results of immunohistochemical staining for SATB2. Ordinary adenocarcinoma of the colon had $29(97 \%)$ positive cases: 2 at $1+, 2$ at $2+$ and 25 at $3+$ (Figure 3A and B). Mucinous adenocarcinoma of the colon had similar results with $26(87 \%)$ cases: 3,4 and 19 cases at $1+, 2+$ and $3+$, respectively. SATB 2 was frequently positive in adenocarcinomas of the digestive organs (stomach (Figure 3C and D), colon, liver, extrahepatic bile duct, gallbladder, pancreas), and it also expressed in a minority of the noncolorectal adenocarcinomas. The sensitivity and specificity of SATB2 for the diagnosis of adenocarcinoma of colorectal origin were $92 \%$ and $71 \%$, respectively. 
Table II. Expression of prostein in 600 carcinomas from various primary sites.

\begin{tabular}{|c|c|c|c|c|c|}
\hline \multirow[t]{2}{*}{ Primary tumor type } & \multicolumn{4}{|c|}{ Immunohistochemical score* } & \multirow[t]{2}{*}{ Positive cases**(\%) } \\
\hline & 0 & $1+$ & $2+$ & $3+$ & \\
\hline Conventional squamous cell carcinoma, larynx $(n=30)$ & 30 & 0 & 0 & 0 & $0(0 \%)$ \\
\hline Squamous cell carcinoma, esophagus $(n=30)$ & 30 & 0 & 0 & 0 & $0(0 \%)$ \\
\hline Tubular adenocarcinoma, stomach $(\mathrm{n}=30)$ & 30 & 0 & 0 & 0 & $0(0 \%)$ \\
\hline Poorly cohensive carcinoma, stomach $(\mathrm{n}=30)$ & 30 & 0 & 0 & 0 & $0(0 \%)$ \\
\hline Ordinary adenocarcinoma, colon $(n=30)$ & 30 & 0 & 0 & 0 & $0(0 \%)$ \\
\hline Mucinous adenocarcinoma, colon $(\mathrm{n}=30)$ & 30 & 0 & 0 & 0 & $0(0 \%)$ \\
\hline Papillary carcinoma, thyroid $(n=30)$ & 30 & 0 & 0 & 0 & $0(0 \%)$ \\
\hline Adenocarcinoma, lung $(\mathrm{n}=30)$ & 30 & 0 & 0 & 0 & $0(0 \%)$ \\
\hline Squamous cell carcinoma, lung $(n=30)$ & 30 & 0 & 0 & 0 & $0(0 \%)$ \\
\hline Classical hepatocellular carcinoma, liver $(n=30)$ & 30 & 0 & 0 & 0 & $0(0 \%)$ \\
\hline Adenocarcinoma, liver $(n=30)$ & 29 & 1 & 0 & 0 & $1(3 \%)$ \\
\hline Adenocarcinoma, extrahepatic bile duct $(n=30)$ & 30 & 0 & 0 & 0 & $0(0 \%)$ \\
\hline Adenocarcinoma, gallbladder $(\mathrm{n}=30)$ & 30 & 0 & 0 & 0 & $0(0 \%)$ \\
\hline Ductal adenocarcinoma, pancreas $(n=30)$ & 30 & 0 & 0 & 0 & $0(0 \%)$ \\
\hline Infiltrating urothelial carcinoma, urinary bladder $(n=30)$ & 30 & 0 & 0 & 0 & $0(0 \%)$ \\
\hline Infiltrating urothelial carcinoma, ureter $(\mathrm{n}=30)$ & 30 & 0 & 0 & 0 & $0(0 \%)$ \\
\hline Acinar adenocarcinoma, prostate $(\mathrm{n}=30)$ & 0 & 0 & 0 & 30 & $30(100 \%)$ \\
\hline Invasive carcinoma of no special type, breast $(n=30)$ & 29 & 0 & 1 & 0 & $1(3 \%)$ \\
\hline Endometrioid carcinoma, uterine corpus $(\mathrm{n}=30)$ & 30 & 0 & 0 & 0 & $0(0 \%)$ \\
\hline Clear cell carcinoma, ovary $(n=30)$ & 30 & 0 & 0 & 0 & $0(0 \%)$ \\
\hline
\end{tabular}

*Score $0: 0 \%$ reactive tumor cells; $1+: 1 \%$ to $10 \%$ reactive tumor cells; $2+: 11 \%$ to $50 \%$ reactive tumor cells and $3+: 51 \%$ to $100 \%$ reactive tumor cells. **Data for any amount of reactivity considered positive.

Table III. Expression of uroplakin II in 600 carcinomas from various primary sites.

\begin{tabular}{|c|c|c|c|c|c|}
\hline \multirow[t]{2}{*}{ Primary tumor type } & \multicolumn{4}{|c|}{ Immunohistochemical score* } & \multirow[t]{2}{*}{ Positive cases**(\%) } \\
\hline & 0 & $1+$ & $2+$ & $3+$ & \\
\hline Conventional squamous cell carcinoma, larynx $(n=30)$ & 30 & 0 & 0 & 0 & $0(0 \%)$ \\
\hline Squamous cell carcinoma, esophagus $(n=30)$ & 30 & 0 & 0 & 0 & $0(0 \%)$ \\
\hline Tubular adenocarcinoma, stomach $(\mathrm{n}=30)$ & 30 & 0 & 0 & 0 & $0(0 \%)$ \\
\hline Poorly cohensive carcinoma, stomach $(\mathrm{n}=30)$ & 30 & 0 & 0 & 0 & $0(0 \%)$ \\
\hline Ordinary adenocarcinoma, colon $(n=30)$ & 29 & 1 & 0 & 0 & $1(3 \%)$ \\
\hline Mucinous adenocarcinoma, colon $(\mathrm{n}=30)$ & 30 & 0 & 0 & 0 & $0(0 \%)$ \\
\hline Papillary carcinoma, thyroid $(n=30)$ & 30 & 0 & 0 & 0 & $0(0 \%)$ \\
\hline Adenocarcinoma, lung $(\mathrm{n}=30)$ & 30 & 0 & 0 & 0 & $0(0 \%)$ \\
\hline Squamous cell carcinoma, lung $(\mathrm{n}=30)$ & 29 & 1 & 0 & 0 & $1(3 \%)$ \\
\hline Classical hepatocellular carcinoma, liver $(n=30)$ & 27 & 3 & 0 & 0 & $3(10 \%)$ \\
\hline Adenocarcinoma, liver $(n=30)$ & 30 & 0 & 0 & 0 & $0(0 \%)$ \\
\hline Adenocarcinoma, extrahepatic bile duct $(n=30)$ & 30 & 0 & 0 & 0 & $0(0 \%)$ \\
\hline Adenocarcinoma, gallbladder $(\mathrm{n}=30)$ & 30 & 0 & 0 & 0 & $0(0 \%)$ \\
\hline Ductal adenocarcinoma, pancreas $(n=30)$ & 30 & 0 & 0 & 0 & $0(0 \%)$ \\
\hline Infiltrating urothelial carcinoma, urinary bladder $(n=30)$ & 14 & 4 & 7 & 5 & $16(53 \%)$ \\
\hline Infiltrating urothelial carcinoma, ureter $(\mathrm{n}=30)$ & 12 & 7 & 7 & 4 & $18(60 \%)$ \\
\hline Acinar adenocarcinoma, prostate $(n=30)$ & 30 & 0 & 0 & 0 & $0(0 \%)$ \\
\hline Invasive carcinoma of no special type, breast $(n=30)$ & 30 & 0 & 0 & 0 & $0(0 \%)$ \\
\hline Endometrioid carcinoma, uterine corpus $(\mathrm{n}=30)$ & 29 & 1 & 0 & 0 & $1(3 \%)$ \\
\hline Clear cell carcinoma, ovary $(n=30)$ & 30 & 0 & 0 & 0 & $0(0 \%)$ \\
\hline
\end{tabular}

*Score $0: 0 \%$ reactive tumor cells; $1+: 1 \%$ to $10 \%$ reactive tumor cells; $2+: 11 \%$ to $50 \%$ reactive tumor cells and $3+: 51 \%$ to $100 \%$ reactive tumor cells. **Data for any amount of reactivity considered positive. 

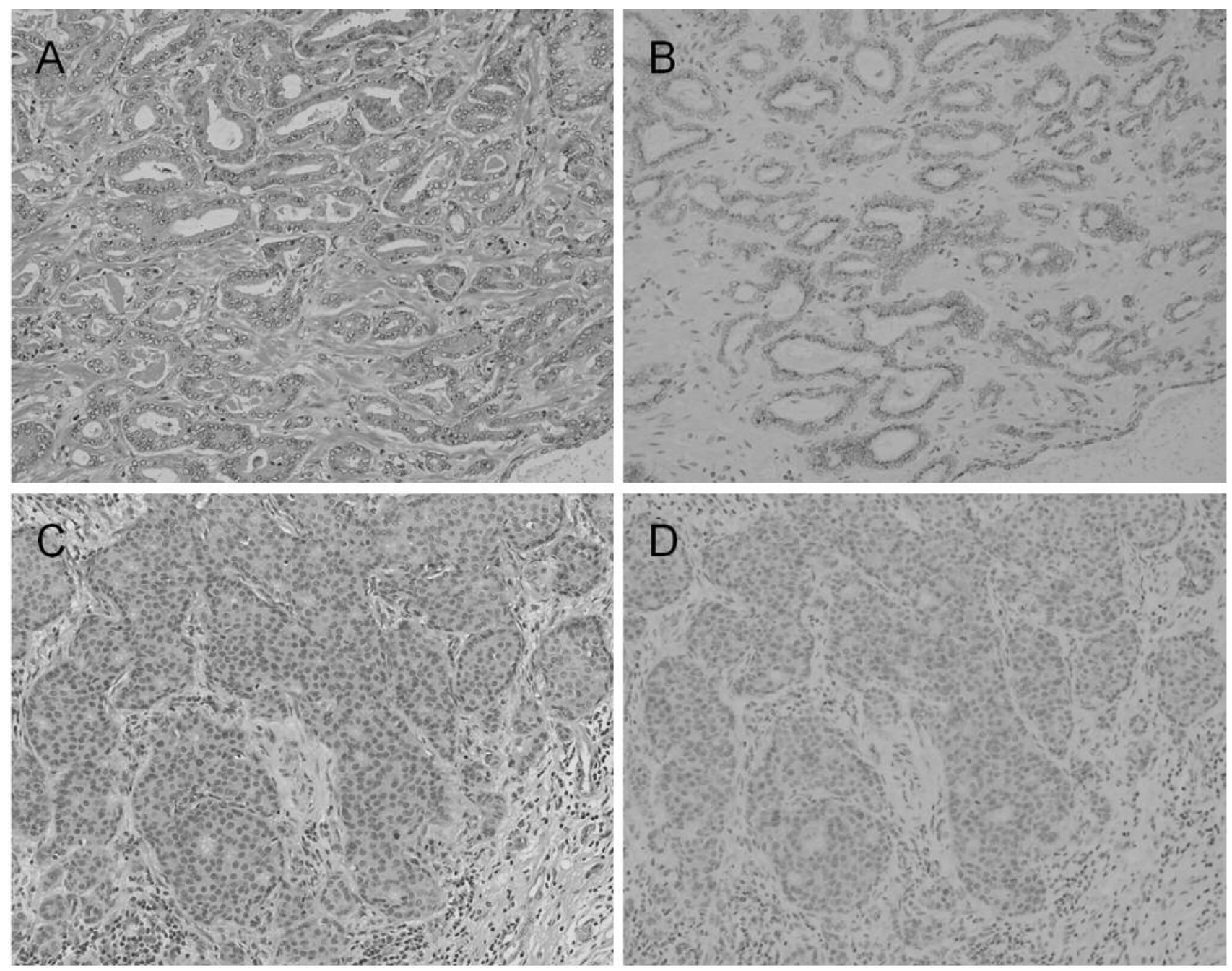

Figure 1. Representative immunohistochemical images of prostein in carcinoma tissues. Acinar adenocarcinoma of the prostate (A) which exhibits prostein immunoreactivity $(B)$ in the cytoplasm with Golgi-type distribution. Invasive carcinoma of no special type of the breast $(C)$ which exhibits prostein immunoreactivity $(D)$ in the cytoplasm. Original magnification $\times 200$.

\section{Discussion}

Immunohistochemistry is often used as a diagnostic tool to determine the primary site of carcinomas of unknown origin. Although a common method for immunohistochemistry uses tissue microarray array (TMA) blocks, we used whole paraffin blocks in our study. Utilizing whole paraffin blocks decreases the chance of false-negative results compared to TMA blocks, which increases the accuracy of our results for prostein, uroplakin II and SATB2 expression.

All prostate adenocarcinomas were immunopositive for prostein with consistently diffuse reactivity. Only rare cases (1 hepatic adenocarcinoma and 1 mammary carcinoma) of the 570 non-prostate carcinomas had labeling for prostein, and it was always focal. Prostein is a highly sensitive and specific marker of prostate adenocarcinomas. Although prostate-specific antigen (PSA) is the most commonly-used immunohistochemical marker for identification of tumors of prostate origin, Sheridan et al. showed that prostein is a more effective marker than PSA (5). They reported that prostein was positive in 2 cases of metastatic adenocarcinoma of the prostate that were PSA-negative. It had no significant background staining and consistently exhibited a perinuclear cytoplasmic staining pattern that was easily interpretable in most cases (5).

The UCs had focal and diffuse immunopositivity for uroplakin II: $53 \%$ of the urinary bladder cases and $60 \%$ of the ureter cases. Only rare cases of the 540 non-urinary 

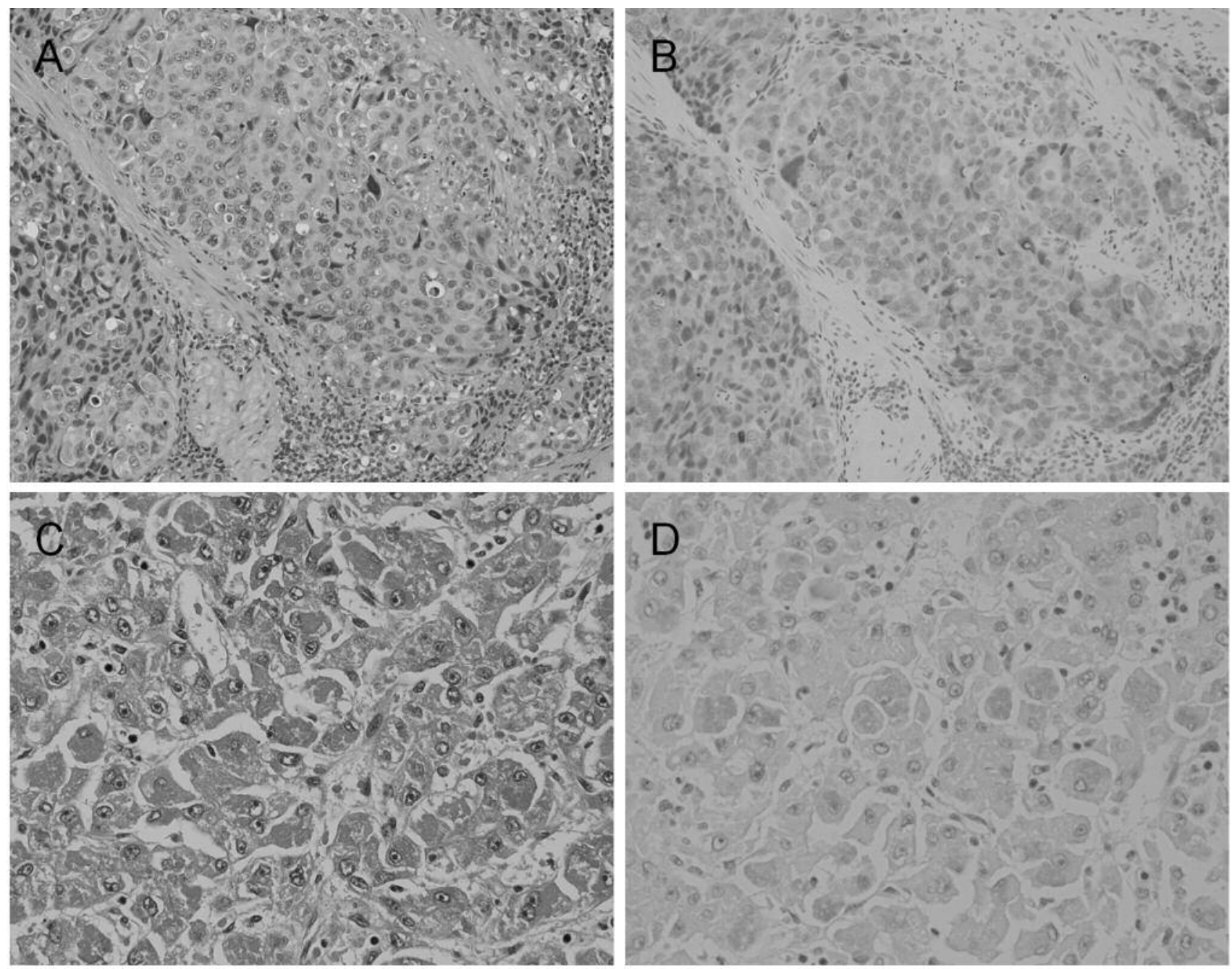

Figure 2. Representative immunohistochemical images of uroplakin II in carcinoma tissues. Infiltrating urothelial carcinoma of the urinary bladder (A) which exhibits uroplakin II immunoreactivity $(B)$ in the cytoplasm. Classical hepatocellular carcinoma of the liver $(C)$ which exhibits uroplakin II immunoreactivity $(D)$ in the intracytoplasmic hyaline bodies. Original magnification $\times 200(A$ and B); $\times 400(C$ and D).

tract carcinomas (1 colorectal adenocarcinoma, 1 pulmonary SCC, 3 hepatic HCCs and 1 uterine EC) of 540 carcinomas of the non-urinary tract had labeling for uroplakin II, and it was always very focal. Uroplakin II is a moderately sensitive and highly specific marker of UCs of the urinary tract. Although immunohistochemical analyses regarding p63, p40, GATA3, and uroplakin III expression are often used to help identify the urothelial origin of UC, p63 and p40 are expressed in almost all SCCs of various organs, and GATA3 is frequently expressed in invasive breast carcinomas $(1,6,16,20,21)$. In addition, Hoang et al. showed that uroplakin II and uroplakin III immunostaining had sensitivities of $79 \%$ and $34-55 \%$ for UCs of the urinary bladder, respectively (6). Li et al. showed by immunohistochemistry that uroplakin II expression was higher than uroplakin III expression in bladder UCs (44\% versus 17\%) and upper urinary tract UCs (67\% versus $46 \%$ ) (16). Furthermore, Smith et al. showed that uroplakin II immunostaining had greater intensity and proportion than uroplakin III in metastatic UCs, consistent with its higher sensitivity (73\% vs. 37\%, respectively) (20). These results indicate that immunohistochemical analysis of uroplakin II is a more valuable marker than uroplakin III for confirming the urothelial origin of carcinomas.

Although CDX2 has been proposed as a colorectal adenocarcinoma immunohistochemical diagnostic marker, it is also frequently expressed in adenocarcinomas of the stomach, the pancreas, the biliary tract and the ovary (1). 

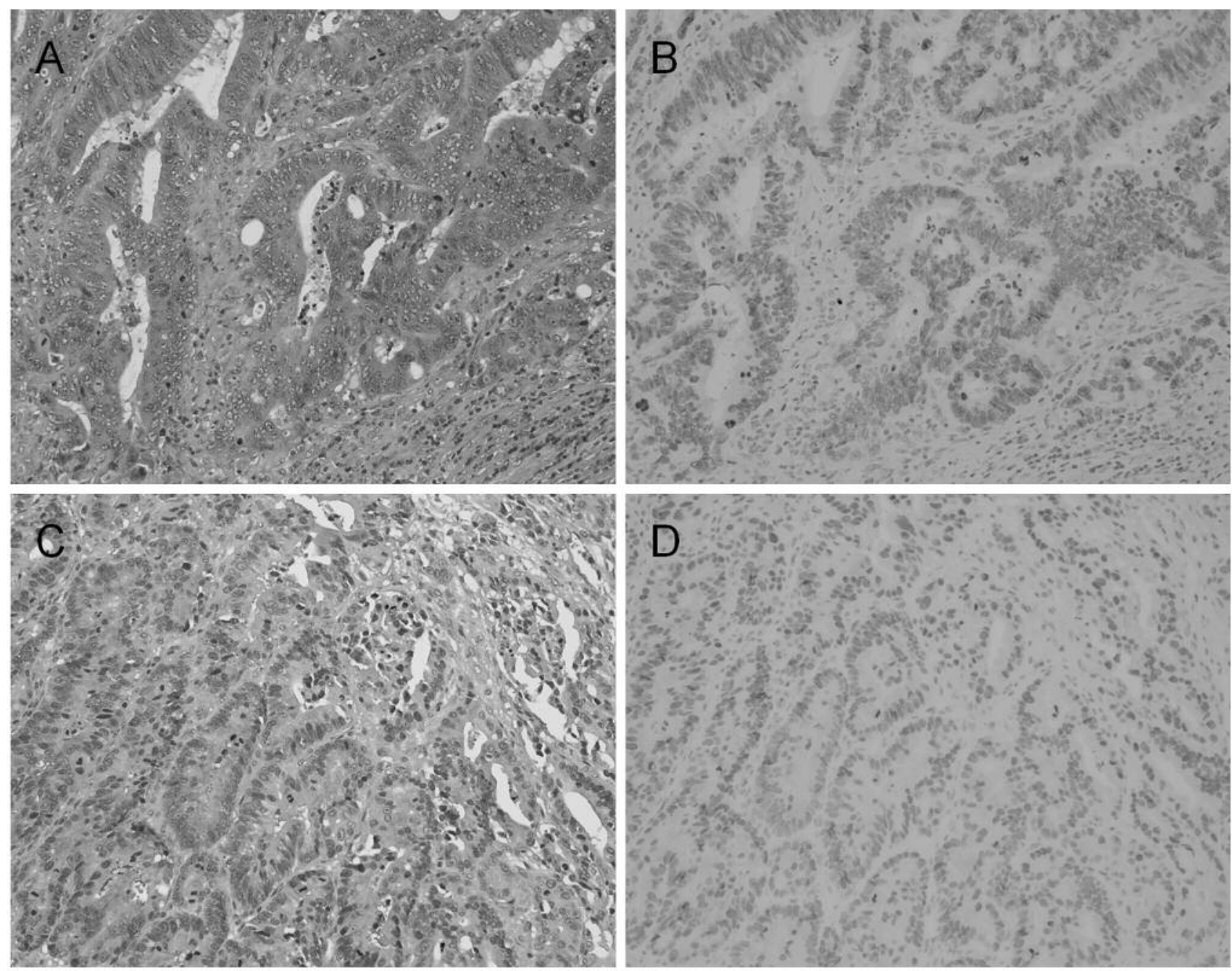

Figure 3. Representative immunohistochemical images of SATB2 in carcinoma tissues. Ordinary adenocarcinoma of the colon (A) which exhibits SATB2 immunoreactivity $(B)$ in the nucleus. Tubular adenocarcinoma of the stomach $(C)$ which exhibits SATB2 immunoreactivity $(D)$ in the nucleus. Original magnification $\times 200$.

Our results showed that SATB2 as well as CDX2 expressed in carcinomas of organs other than the colorectal region (larynx, esophagus, stomach, thyroid, lung, liver, extrahepatic bile duct, gallbladder, pancreas, ureter, prostate, breast, uterine corpus and ovary), and reactivity for SATB2 may be focal or diffuse. In particular, it was frequently expressed in adenocarcinomas of digestive organs. Our results showed that SATB2 may be a highly sensitive marker of colorectal adenocarcinoma but suffered from lower specificity, which suggests that immunostaining for SATB2 can add important information to, at least, rule out adenocarcinomas of colorectal origin.

In conclusion, transcription factors offer superior sensitivity and specificity compared to cytoplasmic markers.
Nevertheless, our results indicate that prostein and uroplakin II are very useful markers for diagnosing the site of origin in carcinomas of unknown primary origin. Meanwhile, SATB2 could add important information to help rule out adenocarcinomas of colorectal origin.

\section{Conflicts of Interest}

None of the Authors have any conflict of interest in regard to this study.

\section{Acknowledgements}

The Authors thank Ms. W. Iha for the technical support. 
Table IV. Expression of SATB2 in 600 carcinomas from various primary sites.

\begin{tabular}{|c|c|c|c|c|c|}
\hline \multirow[t]{2}{*}{ Primary tumor type } & \multicolumn{4}{|c|}{ Immunohistochemical score* } & \multirow[t]{2}{*}{ Positive cases $* *(\%)$} \\
\hline & 0 & $1+$ & $2+$ & $3+$ & \\
\hline Conventional squamous cell carcinoma, larynx $(n=30)$ & 26 & 3 & 1 & 0 & $4(13 \%)$ \\
\hline Squamous cell carcinoma, esophagus $(n=30)$ & 20 & 9 & 1 & 0 & $10(33 \%)$ \\
\hline Tubular adenocarcinoma, stomach $(\mathrm{n}=30)$ & 6 & 10 & 8 & 6 & $24(80 \%)$ \\
\hline Poorly cohensive carcinoma, stomach $(\mathrm{n}=30)$ & 18 & 5 & 6 & 1 & $12(40 \%)$ \\
\hline Ordinary adenocarcinoma, colon $(n=30)$ & 1 & 2 & 2 & 25 & $29(97 \%)$ \\
\hline Mucinous adenocarcinoma, colon $(\mathrm{n}=30)$ & 4 & 3 & 4 & 19 & $26(87 \%)$ \\
\hline Papillary carcinoma, thyroid $(n=30)$ & 25 & 5 & 0 & 0 & $5(17 \%)$ \\
\hline Adenocarcinoma, lung $(\mathrm{n}=30)$ & 25 & 4 & 1 & 0 & $5(17 \%)$ \\
\hline Squamous cell carcinoma, lung $(n=30)$ & 22 & 6 & 2 & 0 & $8(27 \%)$ \\
\hline Classical hepatocellular carcinoma, liver $(n=30)$ & 22 & 4 & 3 & 1 & $8(27 \%)$ \\
\hline Adenocarcinoma, liver $(n=30)$ & 18 & 6 & 4 & 2 & $12(40 \%)$ \\
\hline Adenocarcinoma, extrahepatic bile duct $(n=30)$ & 17 & 8 & 4 & 1 & $13(43 \%)$ \\
\hline Adenocarcinoma, gallbladder $(\mathrm{n}=30)$ & 16 & 10 & 4 & 0 & $14(47 \%)$ \\
\hline Ductal adenocarcinoma, pancreas $(n=30)$ & 25 & 5 & 0 & 0 & $5(17 \%)$ \\
\hline Infiltrating urothelial carcinoma, urinary bladder $(n=30)$ & 30 & 0 & 0 & 0 & $0(0 \%)$ \\
\hline Infiltrating urothelial carcinoma, ureter $(\mathrm{n}=30)$ & 26 & 4 & 0 & 0 & $4(13 \%)$ \\
\hline Acinar adenocarcinoma, prostate $(n=30)$ & 27 & 2 & 1 & 0 & $3(10 \%)$ \\
\hline Invasive carcinoma of no special type, breast $(n=30)$ & 20 & 8 & 1 & 1 & $10(33 \%)$ \\
\hline Endometrioid carcinoma, uterine corpus $(\mathrm{n}=30)$ & 18 & 11 & 0 & 1 & $12(40 \%)$ \\
\hline Clear cell carcinoma, ovary $(n=30)$ & 20 & 10 & 0 & 0 & $10(33 \%)$ \\
\hline
\end{tabular}

*Score $0: 0 \%$ reactive tumor cells; $1+: 1 \%$ to $10 \%$ reactive tumor cells; $2+: 11 \%$ to $50 \%$ reactive tumor cells and $3+: 51 \%$ to $100 \%$ reactive tumor cells. **Data for any amount of reactivity considered positive.

\section{References}

1 Conner JR and Hornick JL: Metastatic carcinoma of unknown primary: diagnostic approach using immunohistochemistry. Adv Anat Pathol 22: 149-167, 2015.

2 Abbruzzese JL, Abbruzzese MC, Lenzi R, Hess KR and Raber $\mathrm{MN}$ : Analysis of a diagnostic strategy for patients with suspected tumors of unknown origin. J Clin Oncol 13: 2094-2103, 1995.

3 Seipel AH, Samaratunga H, Delahunt B, Wiklund P, Clements M and Egevad L: Immunohistochemistry of ductal adenocarcinoma of the prostate and adenocarcinomas of non-prostatic origin: a comparative study. APMIS 124: 263-270, 2016.

4 Miyamoto Y, Suyama K and Baba H: Recent Advances in Targeting the EGFR Signaling Pathway for the Treatment of Metastatic Colorectal Cancer. Int J Mol Sci 18: E752, 2017.

5 Sheridan T, Herawi M, Epstein JI and Illei PB: The role of P501S and PSA in the diagnosis of metastatic adenocarcinoma of the prostate. Am J Surg Pathol 31: 1351-1355, 2007.

6 Hoang LL, Tacha DE, Qi W, Yu C, Bremer RE, Chu J, Haas TS and Cheng L: A newly developed uroplakin II antibody with increased sensitivity in urothelial carcinoma of the bladder. Arch Pathol Lab Med 138: 943-949, 2014.

7 Moh M, Krings G, Ates D, Aysal A, Kim GE and Rabban JT: SATB2 expression distinguishes ovarian metastases of colorectal and appendiceal origin from primary ovarian tumors of mucinous or endometrioid type. Am J Surg Pathol 40: 419-432, 2016.

8 Kalos M, Askaa J, Hylander BL, Repasky EA, Cai F, Vedvick T, Reed SG, Wright GL Jr. and Fanger GR: Prostein expression is highly restricted to normal and malignant prostate tissues. Prostate 60: 246-256, 2004.

9 Chuang AY, DeMarzo AM, Veltri RW, Sharma RB, Bieberich CJ and Epstein JI: Immunohistochemical differentiation of highgrade prostate carcinoma from urothelial carcinoma. Am J Surg Pathol 31: 1246-1255, 2007.

10 Lane Z, Hansel DE and Epstein JI: Immunohistochemical expression of prostatic antigens in adenocarcinoma and villous adenoma of the urinary bladder. Am J Surg Pathol 32: 13221326, 2008.

11 Srinivasan M and Parwani AV: Diagnostic utility of p63/P501S double sequential immunohistochemical staining in differentiating urothelial carcinoma from prostate carcinoma. Diagn Pathol 6: 67, 2011.

12 Magnusson K, de Wit M, Brennan DJ, Johnson LB, McGee SF, Lundberg E, Naicker K, Klinger R, Kampf C, Asplund A, Wester K, Gry M, Bjartell A, Gallagher WM, Rexhepaj E, Kilpinen S, Kallioniemi OP, Belt E, Goos J, Meijer G, Birgisson H, Glimelius B, Borrebaeck CA, Navani S, Uhlén M, O'Connor DP, Jirström $\mathrm{K}$ and Pontén F: SATB2 in combination with cytokeratin 20 identifies over $95 \%$ of all colorectal carcinomas. Am J Surg Pathol 35: 937-948, 2011.

13 Mohanty SK, Smith SC, Chang E, Luthringer DJ, Gown AM, Aron M and Amin MB: Evaluation of contemporary prostate and urothelial lineage biomarkers in a consecutive cohort of poorly differentiated bladder neck carcinomas. Am J Clin Pathol 142: 173-183, 2014.

14 Seipel AH, Samaratunga H, Delahunt B, Wiklund F, Wiklund P, Lindberg J, Grönberg H and Egevad L: Immunohistochemical 
profile of ductal adenocarcinoma of the prostate. Virchows Arch 465: 559-565, 2014.

15 Dragomir A, de Wit M, Johansson C, Uhlen M and Pontén F: The role of SATB2 as a diagnostic marker for tumors of colorectal origin: Results of a pathology-based clinical prospective study. Am J Clin Pathol 141: 630-638, 2014.

16 Li W, Liang Y, Deavers MT, Kamat AM, Matin SF, Dinney CP, Czerniak B and Guo CC: Uroplakin II is a more sensitive immunohistochemical marker than uroplakin III in urothelial carcinoma and its variants. Am J Clin Pathol 142: 864-871, 2014.

17 Tian W, Guner G, Miyamoto H, Cimino-Mathews A, GonzalezRoibon N, Argani P, Li X, Sharma R, Subhawong AP, Rezaei K, Bivalacqua TJ, Epstein JI, Bishop JA and Netto GJ: Utility of uroplakin II expression as a marker of urothelial carcinoma. Hum Pathol 46: 58-64, 2015.

18 Hoang LL, Tacha D, Bremer RE, Haas TS and Cheng L: Uroplakin II (UPII), GATA3, and p40 are highly sensitive markers for the differential diagnosis of invasive urothelial carcinoma. Appl Immunohistochem Mol Morphol 23: 711-716, 2015 .
19 Leivo MZ, Elson PJ, Tacha DE, Delahunt B and Hansel DE: A combination of p40, GATA-3 and uroplakin II shows utility in the diagnosis and prognosis of muscle-invasive urothelial carcinoma. Pathology 48: 543-549, 2016.

20 Smith SC, Mohanty SK, Kunju LP, Chang E, Chung F, Carvalho JC, Paner GP, Hansel DE, Luthringer DJ, de Peralta-Ventrurina $\mathrm{MN}$ and Amin MB: Uroplakin II outperforms uroplakin III in diagnostically challenging settings. Histopathology 65: 132-138, 2014.

21 Cakir A, Isik Gonul I, Ekinci O, Cetin B, Benekli M and Uluoglu O: GATA3 expression and its relationship with clinicopathological parameters in invasive breast carcinomas. Pathol Res Pract 213: 227-234, 2017.

Received June 12, 2018

Revised July 3, 2018

Accepted July 4, 2018 